\title{
Research on Voltage Stability Boundary under Different Reactive Power Control Mode of DFIG Wind Power Plant
}

\author{
Rui Ma ${ }^{\dagger}$, Zeyu Qin*, Wencan Yang* and Mo Li*
}

\begin{abstract}
A novel method is proposed to construct the voltage stability boundary of power system considering different Reactive Power Control Mode (RPCM) of Doubly-Fed Induction Generator (DFIG) Wind Power Plant (WPP). It can be used for reflecting the static stability status of grid operation with wind power penetration. The analytical derivation work of boundary search method can expound the mechanism and parameters relationship of different WPP RPCMs. In order to improve the load margin and find a practical method to assess the voltage security of power system, the approximate method of constructing voltage stability boundary and the critical points search algorithms under different RPCMs of DFIG WPP are explored, which can provide direct and effective reference data for operators.
\end{abstract}

Keywords: Reactive power control, Doubly-fed induction generator, Wind power plant, Load margin, Voltage stability.

\section{Introduction}

As a part of clean energy resource, wind power is developing rapidly and vigorously, but the stability of wind power injection is the key factor restricting the development of large-scale Wind Power Plants (WPPs) connected to grid [1]. To solve this problem, a new voltage stability boundary is constructed with considering wind power injection. In current years, the number of WPP made up with Doubly-Fed Induction Generators (DFIG) is rapidly increasing [2], and the Reactive Power Control Mode (RPCM) of WPP is studied to make the converter of wind turbine as continuous reactive power source for supporting the voltage stability of power system more effectively [3].

In the research field of static stability, the voltage stability boundary of power system can reflect the impact of power system operation state more directly, more comprehensively and more systematically. With the work done in [4-7], quite a few contribution from the research of this boundary can be summarized as follows. As the pioneer presenting the conception of voltage stability boundary, Felix Wu advocated the steady-state security boundary for the first time and defined it as hyper boxes in the power injection space to reflect the operation state of the transmission line power flow, the node voltage and the generator constraints [4]. Along with the development of voltage stability boundary, Ian Dobson further demonstrated

$\dagger$ Corresponding Author: College of Electrical and Information Engineering, Changsha University of Science \& Technology, China. (marui818@126.com)

* College of Electrical and Information Engineering, Changsha University of Science \& Technology, China

(qinzeyu258989231@gmail.com,598248634@qq.com, lee mone@163.com)

Received: August 8, 2015; Accepted: June 18, 2016 that the steady-state voltage boundary was to be depicted as a curved surface consisted of bifurcation points [5]. Afterwards, numerous researches have developed this conception into power system online monitoring application $[6,7]$. After collecting and synthesizing previous scientific work in this research fields, voltage stability boundary is applied in power injection space to analyze the steady state stability of power system with DFIG wind farm.

Recently, the researches of voltage stability boundary with considering wind power injection are also studied to solve the wind energy gird-adaptive technology. The authors of [8] have derived a hyper-plane equation to estimate part of this boundary in the extended full injection space including wind power. However, they only analyzed the macroscopic Total Transfer Capability (TTC) of power system, and neglected to study the detailed wind power injection impact of WPPs, which affects characters of the boundary. The work of [9] has analyzed the impact of uncertainty of wind power on the boundary, but it has not elaborated the influence from the reactive power of WPP. The authors of [10] presented an approach to determining a local voltage stability boundary for power system with WPPs, and compared the loadabilities of boundary with two kinds of WPPs. However, the extended work for analyzing the reason of the boundary differences still remains to be fully elucidated. To investigate the mechanism of the boundary with DFIG WPP, the influence of reactive power of WPPs was analyzed in this paper by monitoring the power system stability in some viewed spaces.

The RPCMs of wind turbine are explored in many previous researches. As the analysis results of [11] demonstrated, large-scale WPPs made up with DFIG can be used as the continuous reactive power source to support 
the voltage stability of power system by taking advantage of their reactive power control capability. In [12], a preventive countermeasure is proposed to improve the voltage stability margin through the management of the reactive power and its reserve. Moreover, the authors of [13] specified two kinds of reactive power control ancillary services of WPP, which demand that the distributed WPPs do not have to always provide the service of voltage control but must control output power factor in order to maintain the system stability. The scientific results of [14] have also find the way to improve the voltage stability margin, but their research focuses on locating and sizing DG units to achieve their ultimate goals. Furthermore, the research of [15] presented a steady state operation DFIG math model. On the basis of [15], this DFIG math model is applied in this paper and different operation modes are simulated within this voltage stability boundary. The work of mathematical derivation also has been done to explain the different impact of RPCMs within the boundary.

The contribution in this paper can be summarized into the following three main points:

1) The analytical derivations of voltage stability boundary are obtained to study the different impact of RPCMs.

2) Constructing the approximate voltage stability boundary by tangent planes algorithm in complicated systems, and using Continuation Power Flow (CPF) to search the critical points under different RPCMs on boundary.

3) The reactive injection power of DFIG WPP is analyzed to find the characteristics of different RPCMs.

The remainder of this paper is organized in the following sections. In section II, the analytical derivations and computer calculation methods of constructing voltage stability boundary are studied, and the search methods under different wind farm RPCMs are denoted. Section III presents the reactive power analysis of power system with DFIG wind farms. In section IV, the case study of this paper is done. Section V presents the conclusion of this paper.

\section{Voltage Stability Boundary}

The reactive power operation modes of DFIG are voltage control mode and power factor control mode [1618].

Most WPPs usually operate at constant power factor mode in a conservative way, and cannot make full use of reactive power adjustment capability [19]. However, when the DFIG WPPs are connected with grid to execute voltage control mode, they can adjust the voltage and support the stability of system [20].

Thus, to explore the detailed effect of DFIG wind farm control mode, the loadability of voltage stability boundary under different control mode is analyzed by the following methods in this section.

\subsection{Analytical derivation in simple system}

To explore the different detailed effect on load margin between these two kinds of WPP RPCMs, voltage stability boundary is utilized to explain it in a simple system.

Here the wind power injection bus was analyzed to describe the reactive power adjustment of WPP. Assumed that the wind speed is satisfied with Weibull distribution in WPP, the relationship between the WPP injection power $P_{W}$ and wind speed $v$ can be stated as follows.

$$
P_{W}=\left\{\begin{array}{c}
0 \quad v<v_{\text {cut-in }} \text { or } \quad v>v_{\text {cut-out }} \\
\frac{v^{3}-v_{\text {cut-in }}^{3}-v_{\text {- }}^{3}}{v_{\text {cut }- \text { out }}^{3}-v_{\text {cut-in }}^{3}} P_{\text {rated }} \quad v_{\text {cut }- \text { in }} \leq v \leq v_{\text {rated }} \\
P_{\text {rated }} \quad v_{\text {rated }}<v<v_{\text {cut-out }}
\end{array}\right.
$$

The relationship between the variables of this system can be established from Fig. 1. A group of simple power flow equations are denoted as follows.

$$
\left\{\begin{array}{c}
P=P_{L}-P_{W}=\frac{E V}{X} \sin \sigma \\
Q=Q_{L}-Q_{W}=\frac{E V}{X} \cos \sigma-\frac{V^{2}}{X}
\end{array}\right.
$$

After eliminating parameter $\sigma$ of the power flow equation, the model is simplified, and this formula can derive (3).

$$
F=\left(\frac{V^{2}}{X}+Q\right)^{2}+\left(P_{L}-P_{W}\right)^{2}-\left(\frac{E V}{X}\right)^{2}=0
$$

To find the boundary of this system, this power flow equation has to satisfy along with the requirement below.

$$
\operatorname{det}\left(\frac{\partial F}{\partial V}\right)=0
$$

The voltage stability boundary of this system can be illustrated by (5).

$$
V_{\text {boundary }}=\sqrt{\frac{E^{2}}{4}+\frac{X^{2}}{E^{2}}\left(P_{L}-P_{W}\right)^{2}}
$$

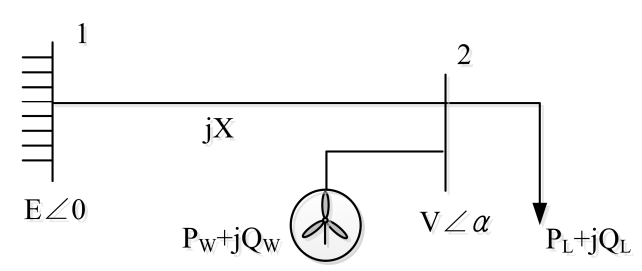

Fig. 1 Simple system with wind power injection. 
When WPP is under power factor control mode, the maximum loadability of this power system can be calculated by (6).

$$
\begin{aligned}
P_{L \cdot \max }= & \sqrt{\frac{E^{4}}{4 X^{2}}\left(1+\tan ^{2} \varphi_{L}\right)+\frac{E}{X}\left(\tan \varphi_{W}-\tan \varphi_{L}\right) P_{W}} \\
& +P_{W}-\frac{E^{2}}{2 X} \tan \varphi_{L}
\end{aligned}
$$

When the WPP is a constant voltage support source at bus 2 , the load margin is deduced by (7).

$$
P_{L \text { max }}=\sqrt{\frac{4 E^{2} V^{2}-E^{4}}{4 X^{2}}}+P_{W}
$$

\subsection{Constructing voltage stability boundary under different RPCMs of DFIG WPP in practical power system}

To study the impact of RPCM on voltage stability boundary in large-scale wind power injection system, CPF method is utilized to search the boundary. By referring to the work of [21] and [22], a novel tangent plane considering DFIG WPPs is used to construct the approximate boundary, and the operation points under different WPP RPCMs are also simulated to analyze static security of power system.

\subsubsection{The CPF Math Model of Power System with DFIG WPP}

$f_{W}=\left[\begin{array}{c}V_{s} \cos \theta_{s}+R_{s} I_{s} \cos \phi_{s}-\left(X_{s}+X_{m}\right) I_{s} \sin \phi_{s}+X_{m} I_{r} \sin \phi_{r}=0 \\ V_{s} \sin \theta_{s}-R_{s} I_{s} \sin \phi_{s}+\left(X_{s}+X_{m}\right) I_{s} \cos \phi_{s}-X_{m} I_{r} \cos \phi_{r}=0 \\ V_{r} \cos \theta_{r}-s X_{m} I_{s} \sin \phi_{s}-R_{r} I_{r} \cos \phi_{r}+s\left(X_{s}+X_{m}\right) I_{r} \sin \phi_{r}=0 \\ V_{r} \sin \theta_{r}+s X_{m} I_{s} \cos \phi_{s}-R_{r} I_{r} \sin \phi_{r}-s\left(X_{s}+X_{m}\right) I_{r} \cos \phi_{r}=0 \\ P_{e}-V_{s} I_{s} \cos \left(\theta_{s}-\phi_{s}\right)+V_{r} I_{r} \cos \left(\theta_{r}-\phi_{r}\right)=0 \\ Q_{e}-V_{s} I_{s} \sin \left(\theta_{s}-\phi_{s}\right)=0 \\ P_{e}+I_{s}^{2} R_{s}+I_{r}^{2} R_{r}+P_{G B, L s s s}-k_{0}(1-s)^{3}=0\end{array}\right]$

The math model of DFIG can be expressed by $f_{W}$. In order to construct the steady state math model of DFIG, $V_{r}$, $\theta_{r}, I_{s}, \phi_{s}, I_{r}, \phi_{r}, s$ should be the steady state parameters, which are also the solution of the math model. To describe the injection power of WPP, denote the injection power of i-th DFIG WPP as $P_{W i}$ by accumulating the electrical power $P_{e}$ of each DFIG wind turbine.

On the basis of [15], math model of DFIG WPP is added to the parametric power flow equations for the first time. The parametric power flow equations can be written as follows, which has considered the constraint relationship of WPP RPCM.

In (9), $f_{C}$ represents equations of DFIG WPP reactive compensation control mode. When the RPCM of DFIG
WPP is power factor control mode, $f_{C l}$ will be chosen as a part of CPF calculation math model. $f_{C l}$ represents the voltage control mode equations of DFIG WPP.

$$
\left\{\begin{array}{c}
f_{P}=P_{G i}-P_{L i}+\left(\alpha_{i} \cdot \sum_{j \in N_{G}} P_{G j}\right) \cdot \Delta \lambda-\left(\beta_{i} \cdot \sum_{j \in N_{L}} P_{L j}\right) \cdot \Delta \lambda+P_{W i} \\
-V_{i} \sum_{j \in N_{G} \cup N_{L} \cup N_{W}} V_{j}\left(G_{i j} \cos \theta_{i j}+B_{i j} \sin \theta_{i j}\right)=0 \\
f_{Q}=-Q_{L i}-\left(\beta_{i} \cdot \sum_{j \in N_{L}} Q_{L j}\right) \cdot \Delta \lambda \\
\quad-V_{L i} \sum_{j \in N_{G} \cup N_{L} \cup N_{W}} V_{j}\left(G_{i j} \sin \theta_{i j}-B_{i j} \cos \theta_{i j}\right)=0 \\
f_{C 1}=P \cdot \tan \varphi_{W \cdot r e f}-V_{W i} \sum_{j \in N_{G} \cup N_{L} \cup N_{W}} V_{j}\left(G_{W j} \sin \theta_{W j}-B_{W j} \cos \theta_{W j}\right)=0 \\
\text { or } f_{C 2}=V_{W \cdot r e f}-V_{W i}=0
\end{array}\right.
$$

The CPF math model can be obtained by combining (8) and (9) together, and the equations of this model can be expressed simply as follows:

$$
f\left(x, \lambda, \alpha, \beta, P_{W}\right)=\left[\begin{array}{c}
f_{W} \\
f_{P} \\
f_{Q} \\
f_{C}
\end{array}\right]
$$

\subsubsection{Constructing the Approximate Voltage Stability Boundary under Different RPCMs of DFIG WPP}

To construct the approximate boundary, (10) must be linearized when the operation point is at the critical state. The linearized model is shown in the following:

$$
\begin{gathered}
J_{\text {Power__System }_{1}}\left|* \Delta x+\frac{\partial f}{\partial \lambda}\right| * \Delta \lambda+\frac{\partial f}{\partial \alpha}\left|* \Delta \alpha+\frac{\partial f}{\partial \beta}\right| * \Delta \beta \\
+\frac{\partial f}{\partial P_{W}} \mid * \Delta P_{W}=0
\end{gathered}
$$

The steady state variables $V i, \theta i, V r, \theta r, I s, \phi s, I r, \phi r, s$ are denoted as $x$ in (11), $J_{\text {Power System }}$ is the steady state part of the Jacobian matrix calculated by (10). $\lambda, \alpha, \beta, P_{W}$ are the expanded variables of the parametric power flow model. The expanded parts of this Jacobian matrix are $\partial f / \partial \lambda, \partial f / \partial \alpha$, $\partial f / \partial \beta, \partial f / \partial P_{W}$

To research the Jacobian matrix with adding DFIG steady state model, the part of matrix depicting the DFIG wind farm is shown as follow, which is denoted as $J_{\text {DFIG_Wind_Farm. }}$.

The interrelated matrix between power system and DFIG wind farms can be denoted as $J_{\text {Wind Farm Connection. In this }}$ matrix, $m$ represents the wind farm connected node. Assumed that the power system has $n$ nodes, the number of PQ nodes is $p$, and the number of PV nodes is $q$, which are satisfied with $p+q+1=n$. 


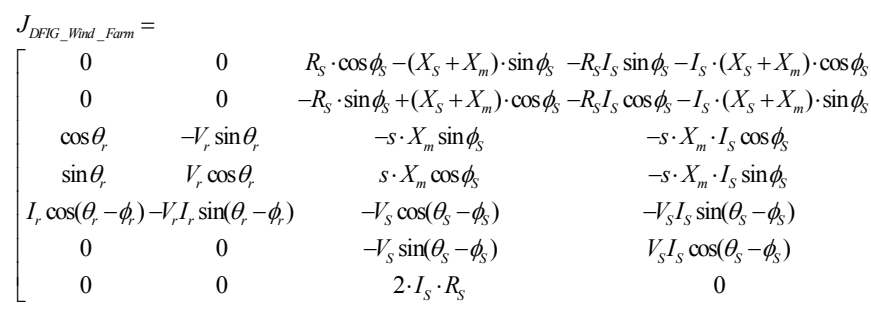

When the DFIG wind farm is operating in power factor control mode, the interrelated matrix of WPP are denoted as follows.

$$
\begin{aligned}
& J_{\text {Wind_Farm_Connection }}= \\
& {\left[\begin{array}{cccc}
\overbrace{0 \cdots 0}^{2(m-1)} & \cos \theta_{S} & \\
V_{S} \cos \theta_{S} & \sin \theta_{S} & \\
& 0 & 0 & 0 \\
& 0 & 0 & 0 \\
V_{S} I_{S} \sin \left(\theta_{S}-\phi_{S}\right) & -I_{S} \cos \left(\theta_{S}-\phi_{S}\right) \\
& -V_{S} I_{S} \cos \left(\theta_{S}-\phi_{S}\right) & -I_{S} \sin \left(\theta_{S}-\phi_{S}\right) \\
& 0 & 0
\end{array}\right]}
\end{aligned}
$$

The interrelated matrix of wind farm under voltage control mode is given in the following matrix:

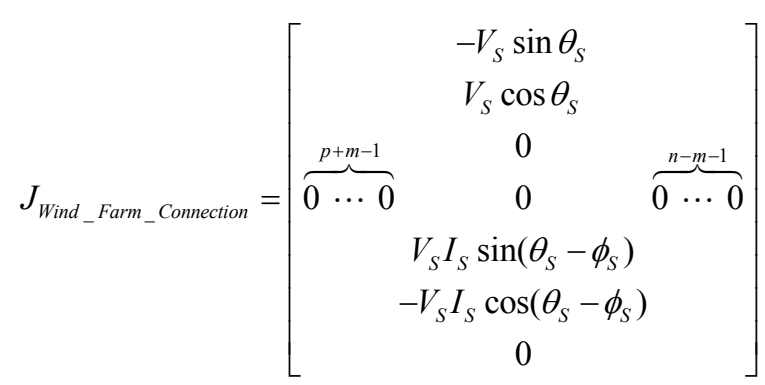

The steady state part of Jacobian matrix $J_{\text {Power_System }}$ consists of 4 parts, which can be expressed below:

$$
J_{\text {Power_System }_{\text {S }}}=\left[\begin{array}{c:c}
\frac{\partial f P}{\partial \theta} \frac{\partial f P}{\partial V} & 0 \\
\frac{\partial f Q}{\partial \theta} \frac{\partial f Q}{\partial V} & \\
\hdashline J_{\text {Wind_Farm_Connection }} & J_{\text {DFIG_Wind_Farm }}
\end{array}\right]
$$

The left eigenvector $\omega$, which deduced from the eigenvalue zero of the Jacobian matrix, should premultiply (11), and then a new equation can be got.

$$
\begin{aligned}
& \omega \cdot J_{\text {Power__system }}\left|* \cdot \Delta x+\omega \cdot \frac{\partial f}{\partial \lambda}\right| * \Delta \lambda+\omega \cdot \frac{\partial f}{\partial \alpha}\left|* \Delta \alpha+\omega \cdot \frac{\partial f}{\partial \beta}\right| \\
& * \Delta \beta+\omega \cdot \frac{\partial f}{\partial P_{W}} \mid * \Delta P_{W}=0
\end{aligned}
$$

Where ' $*$ ' means that the operation state is at the critical state in power system, and $\omega^{\bullet} J_{\text {Power_System }^{*}}{ }^{*}$ are zero in (16). The expression in (16) can be simplified into (17).

$\omega \cdot \frac{\partial f}{\partial \lambda}\left|* \Delta \lambda+\omega \cdot \frac{\partial f}{\partial \alpha}\right| * \Delta \alpha+\omega \cdot \frac{\partial f}{\partial \beta}\left|* \Delta \beta+\omega \cdot \frac{\partial f}{\partial P_{W}}\right| * \Delta P_{W}=0$

The tangent plane equations of voltage stability boundary in $\lambda-\alpha-\beta-P_{W}$ space, which show in (17), can be mapped into $\lambda-P_{G}-P_{L}-P_{W}$ space.

After the critical point location $\left(\left.\lambda\right|_{*} ^{1},\left.P_{G}\right|_{*} ^{1},\left.P_{L}\right|_{*} ^{1},\left.P_{W}\right|_{*} ^{1}\right)$ and its normal vector $\left(K_{\lambda}, K_{G}, K_{L}, K_{W}\right)$ are obtained by using CPF method, the tangent plane expression of this critical point is illustrated below. The voltage stability boundary is fitted by these tangent planes together.

$$
\begin{gathered}
K_{\lambda}\left(\left.\lambda\right|_{*}-\left.\lambda\right|_{*} ^{1}\right)+K_{G}\left(\left.P_{G}\right|_{*}-\left.P_{G}\right|_{*} ^{1}\right)+K_{L} \cdot\left(\left.P_{L}\right|_{*}-\left.P_{L}\right|_{*} ^{1}\right) \\
+K_{W} \cdot\left(\left.P_{W}\right|_{*}-\left.P_{W}\right|_{*} ^{1}\right)=0
\end{gathered}
$$

The different RPCM can lead to different critical points and different normal vectors. The effect from different WPP RPCMs on critical points and loadability $\lambda$ is mainly caused by the different math model of CPF calculation equations (10) and the different construction of power system Jacobian matrix (15).

To clarify the process of voltage stability boundary approximate calculation method clearer, the process is presented below.

\section{The Reactive Compensation Capacity Analysis}

In DFIG WPP, the converter of DFIG, SVC and STATCOM can adjust the reactive injection power of WPP. In this secondary section, the reactive compensation 


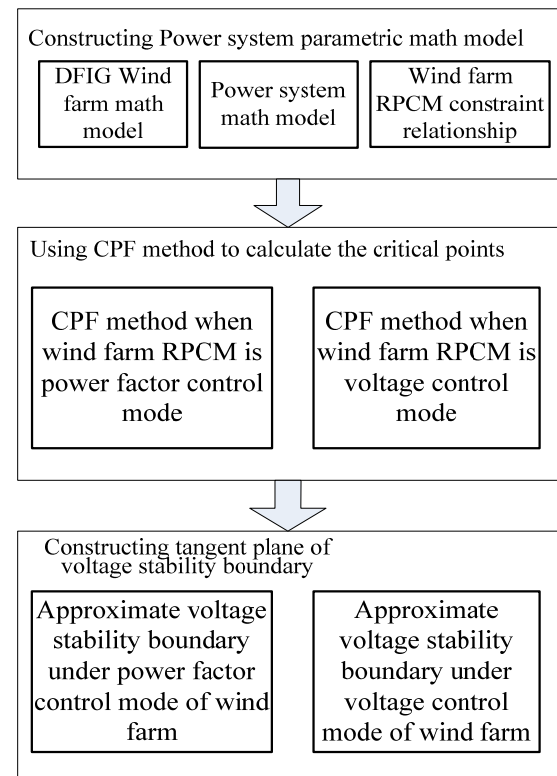

Fig. 2. Algorithm flow chart of constructing the voltage stability boundary.

capacity of WPP is calculated under different RPCM to analyze the voltage stability boundary.

When the operation of WPP use power factor control mode, the WPPs are viewed as PQ nodes to connect the grid. The reactive power constraint equations under power factor control mode are shown as follows.

$$
Q_{W}=P_{W} \tan \varphi_{W . r e f}
$$

When the control mode is under constant voltage, the WPPs are connected with grid as PV nodes, and the calculate formulas of reactive power are expressed as follows,

$$
\begin{gathered}
Q_{W}=\varepsilon \cdot V_{W . r e f} \\
\varepsilon=\sum_{j \in N_{L} \cup N_{G} \cup N_{W}} V_{j}\left(G_{W j} \sin \theta_{W j}-B_{W j} \cos \theta_{W j}\right)
\end{gathered}
$$

Here subscript $W$ is used to represent the index of WPPs.

\section{Case Study}

\subsection{Two buses system with DFIG WPP}

This example examined the analytical derivation of different RPCMs of DFIG WPP within voltage stability boundary. Assumed that the shape coefficient $k$ is 2.0 and scale coefficient $c$ is 10.0, the wind speed of WPP is satisfied with Weibull distribution and the wind power injection $P_{W}$ is fluctuating between $0-4$.0p.u. . The detailed system parameters of Fig. 1 are given in Table 1.
Table 1. Parameters of two bus power system with WPP

\begin{tabular}{c|c|c|c}
\hline Parameters & Values or Ranges & Parameters & Values or Ranges \\
\hline$E$ & $1.00 \mathrm{p} . \mathrm{u}$. & $P_{\text {rated }}$ & $4.00 \mathrm{p} . \mathrm{u}$. \\
\hline $\mathrm{X}$ & $0.1 \mathrm{p} . \mathrm{u}$. & $v_{\text {rated }}$ & $13 \mathrm{~m} / \mathrm{s}$ \\
\hline$v_{\text {cut-in }}$ & $4 \mathrm{~m} / \mathrm{s}$ & $v_{\text {cut-out }}$ & $21 \mathrm{~m} / \mathrm{s}$ \\
\hline$\varphi_{\text {Wref }}$ & - arccos $0.95-\arccos 0.95$ & $V_{\text {ref }}$ & $0.95-1.00 \mathrm{p} . \mathrm{u}$. \\
\hline
\end{tabular}

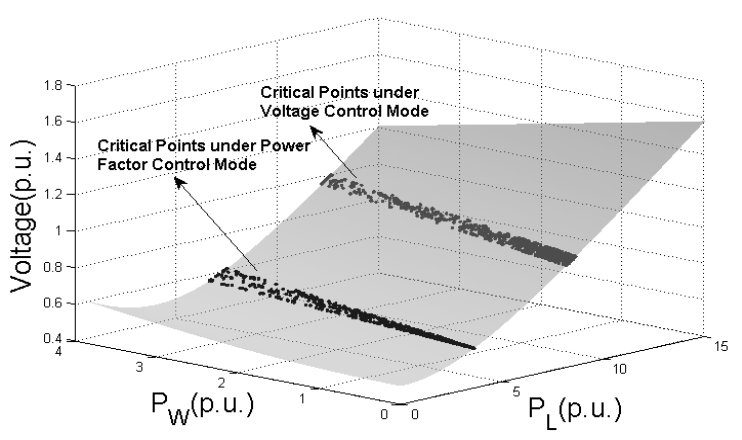

Fig. 3. Voltage stability boundary and critical points under different RPCM.

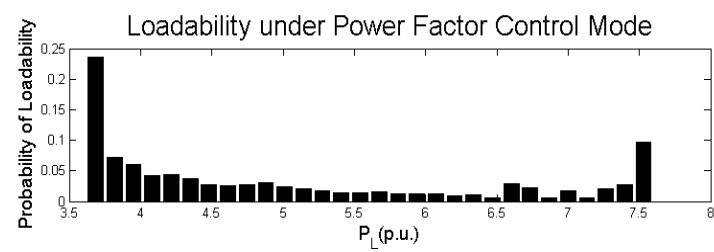

(a)

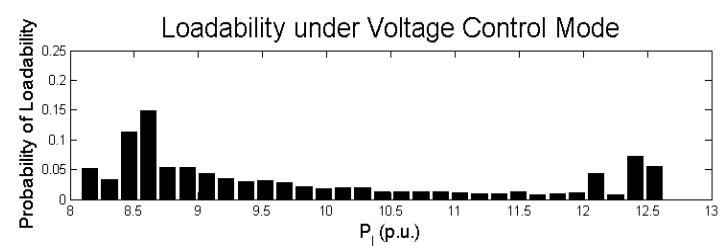

(b)

Fig. 4. Probability distribution of loadability: (a) Under power factor control mode; (b) Under voltage control mode.

When the WPP is operated under power factor control mode, the bound of $\varphi \mathrm{W}$ ref is set between $[-\arccos 0.95$, $\arccos 0.95]$, so the power factor of WPP net can be controlled in $0.95-1.00$. When the voltage control mode of WPP is executed to support the wind injection stability, the voltage reference Vref of WPP is chosen within the range of [0.95p.u., 1.00p.u.], and voltage of WPP can be controlled by putting the reactive power regulators into operation, such as SVC, STATCOM, etc.

By using analytical derivation to compute case study A, the boundary of voltage stability and critical points under different RPCMs are shown in Fig. 1. The points on the boundary are separated into two groups by implementing the different RPCMs in this $V-P_{L^{-}} P_{W}$ space. To analyze the data of critical points under different RPCM, 
$Q_{W}$ under Power Factor Control Mode

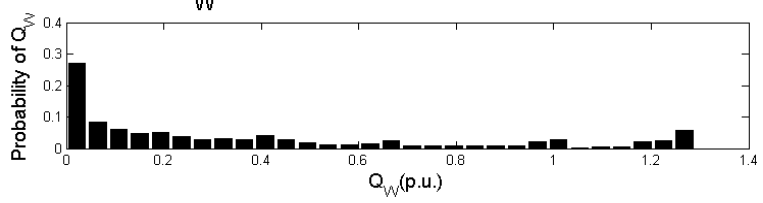

(a)

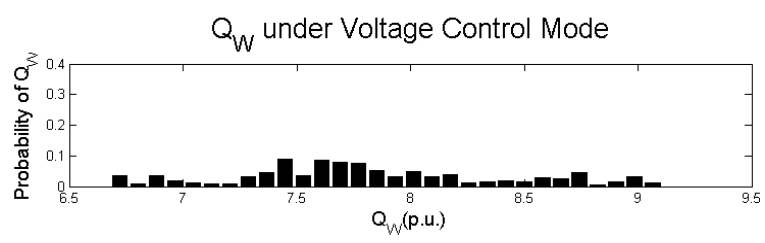

(b)

Fig. 5. Probability distribution of WPP Reactive power: (a) Under power factor control mode; (b) Under voltage control mode.

Table 2. Analysis results of case study A

\begin{tabular}{c|c|c}
\hline RPCM of WPP & $\begin{array}{c}\text { Expectation } \\
\text { Values of } P_{\text {Lcrt }}\end{array}$ & $\begin{array}{c}\text { Expectation } \\
\text { Values of } Q_{\text {Wrt }}\end{array}$ \\
\hline Power Factor Control Mode & 4.9400 p.u. & 0.3340 p.u. \\
\hline Voltage Control Mode & 9.8324 p.u. & 7.8610 p.u. \\
\hline
\end{tabular}

the probability distributions of $P_{L . c r t}$ and $Q_{W}$ are shown in Fig. 4 and Fig. 5, respectively, and the expectation values of $P_{L . c r t}$ and $Q_{W}$ are also shown in Table 2 .

According to the analysis data above, the security adjustment capability of these two kinds of RPCMs can be compared apparently. The loadability under voltage control mode is 9.8324p.u., as well as the expectation value of $P_{L . c r t}$ under power factor control mode is only 4.9400 p.u. . By comparing the expectation value of $P_{L . c r t}$, it is a clear vision that the loadability under voltage control mode is higher than that under power factor control mode. Moreover, to maintain the voltage within the bound, the reactive power regulators are putted into operation, so the expectation values of $Q_{W}$ under voltage control mode is much more than that under the other RPCM.

\subsection{IEEE(New England) 39 nodes system with DFIG WPP}

This case study is based on IEEE (New England) 39 nodes system to analyze the RPCM of large-scale WPP within voltage stability boundary. In this case study, it is assumed that the DFIG WPP is connected to bus 37. The WPP of this power system consists of 518 doubly-fed induction generators, the injection active power and reactive compensation capacity of WPP are 1260MW and 720Mvar respectively. By referring to [9] and using power tracing method to determine the closest generators of WPP, bus 30 and bus 39 are used as connecting compensation generators to balance the fluctuation of wind turbine output. The parameters of DFIG WPP, which are adopted in this

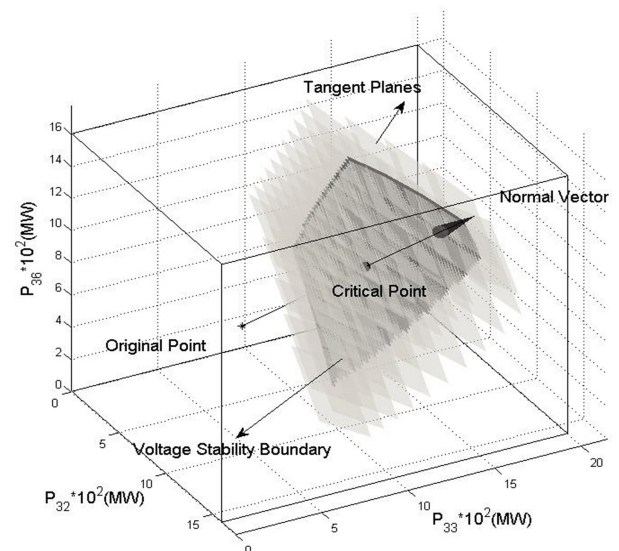

Fig. 6. Approximate voltage stability boundary in $P G_{36^{-}}$ $P G_{32}-P G_{33}$ space.

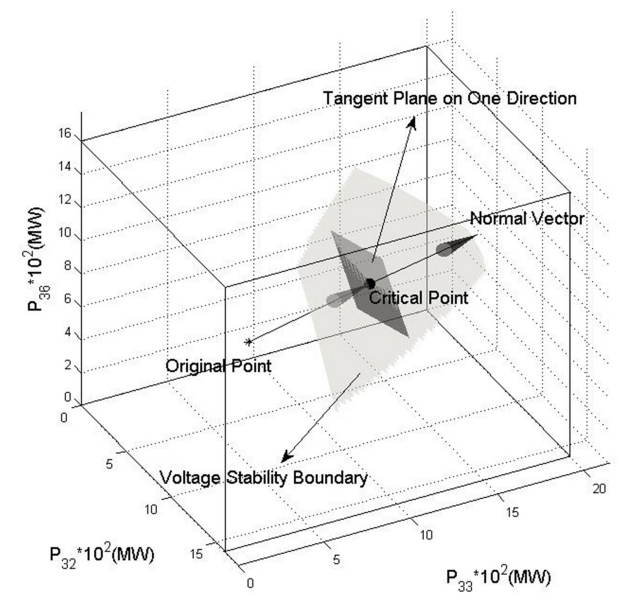

Fig. 7. Tangent plane of approximate boundary in $P G_{36^{-}}$ $P G_{32}-P G_{33}$ space.

case study, can be found in [23].

According to the parameters above, built programs are used to calculate this example. By adopting CPF method, the voltage stability boundary is drawn out. This boundary is constructed in the $P G_{32}-P G_{33}-P G_{36}$ space to monitor the operation and the static stability of power system. To explain the different influences of these two RPCMs of WPP, the operation loadabilities within voltage stability boundary are compared and the reactive power injection of WPP is analyzed.

Fig. 6 shows the approximate boundary of voltage stability boundary accumulated by tangent planes. Traditional power system security analytical method is to search each point in parameter space of power system, but it cannot bring enough information of whole system security. For constructing the voltage stability boundary faster and obtaining more stability information in practical application, tangent plane method is implemented to build the boundary.

In Fig. 7, the single tangent plane of voltage stability boundary is taken out from the approximate boundary 
Table 3. Coordinate data of critical points and normal vectors (Coefficients of corresponding tangent planes) under different reactive power control mode

\begin{tabular}{c|c|c|c|c}
\hline $\begin{array}{c}\text { Wind speed } \\
(\mathrm{m} / \mathrm{s})\end{array}$ & $\begin{array}{c}\text { Critical Point } z_{l} \text { under power factor } \\
\text { control mode }\end{array}$ & $\begin{array}{c}\text { Normal vector (Coefficients of } \\
\text { tangent plane) under power } \\
\text { factor control mode }\end{array}$ & $\begin{array}{c}\text { Critical point } z_{2} \text { under voltage } \\
\text { control mode }\end{array}$ & $\begin{array}{c}\text { Normal vector (Coefficients } \\
\text { of tangent plane) under } \\
\text { voltage control mode }\end{array}$ \\
\hline $0-4,>21$ & {$[1111.12,1093.12,1035.88](\mathrm{MW})$} & {$[0.6150,0.5200,0.5928]$} & {$[1128.50,1110.50,1053.81](\mathrm{MW})$} & {$[0.5962,0.5310,0.6021]$} \\
\hline 5 & {$[1112.01,1094.01,1036.80](\mathrm{MW})$} & {$[0.6137,0.5528,0.5917]$} & {$[1128.76,1110.76,1054.08](\mathrm{MW})$} & {$[0.5955,0.5325,0.6015]$} \\
\hline 7 & {$[1114.56,1096.56,1039.43](\mathrm{MW})$} & {$[0.6102,0.5291,0.5897]$} & {$[1129.43,1111.43,1054.78](\mathrm{MW})$} & {$[0.5937,0.5363,0.5999]$} \\
\hline 8 & {$[1115.92,1097.92,1040.83](\mathrm{MW})$} & {$[0.6084,0.5327,0.5883]$} & {$[1129.68,1111.68,1055.03](\mathrm{MW})$} & {$[0.5923,0.5396,0.5983]$} \\
\hline 11 & {$[1115.68,1097.68,1040.59](\mathrm{MW})$} & {$[0.6072,0.5430,0.5801]$} & {$[1127.70,1109.70,1052.98](\mathrm{MW})$} & {$[0.5907,0.5478,0.5925]$} \\
\hline $13-21$ & {$[1097.23,1079.23,1021.55](\mathrm{MW})$} & {$[0.6282,0.5484,0.5519]$} & {$[1119.52,1101.52,1044.55](\mathrm{MW})$} & {$[0.5974,0.5451,0.5882]$} \\
\hline
\end{tabular}

separately to show in this space, and the operation status moves from original point to critical point.

To master more information of the boundary, the normal vector, i.e. coefficients of tangent planes, is used in the boundary calculation. Data from Fig. 8 and Table 3 show the load margins under different RPCMs within the voltage stability boundary. From the table above, when wind speed is varying, the load margin is changed slightly. For example, critical point $z_{1}$ moves from [1111.12, 1093.12, 1035.88] MW to [1115.68, 1097.68, 1040.59] MW, when wind speed varies from $0 \mathrm{~m} / \mathrm{s}$ to $11 \mathrm{~m} / \mathrm{s}$. The other point $\mathrm{z}_{2}$ is similarly with slight change. However, when the reactive power control mode is switched from voltage control mode to power factor control mode, the load margin will have obvious change, e. g., the critical point moves from $[1115.92,1097.92,1040.83]$ MW to $[1129.68,1111.68$, 1055.03] MW, when wind speed is $8 \mathrm{~m} / \mathrm{s}$ and the control mode is changed. Moreover, when wind speed is from $11 \mathrm{~m} / \mathrm{s}$ to $21 \mathrm{~m} / \mathrm{s}$, the variation range of load margin is also obvious, but it doesn't move as long as the variation range caused by RPCM. From these analyses above, the changes of load margin under different RPCMs are obvious, and these changes are occurred in the same voltage stability boundary in multidimensional space.

In Fig. 9, the voltage stability boundary is shown in the 3-dimensions space which consists of WPP $P W_{37}$, compensation generator $P G_{30}$ and load parameter $\lambda$. The compensation generator $P G_{30}$ and $P G_{39}$ can adjust the fluctuation power injected by WPP in this case. In this figure, the load margins are compared under different RPCMs of WPP, which depict that the load margin under voltage control mode is greater than the other by setting $V_{\text {ref }}=1.00$ p.u. . When the wind turbine is operating in the power factor control mode, the output of WPP reactive power cannot maintain the stability of this area effectively. On this condition, to protect the electrical equipment from damage, the voltage adjust ability of DFIG WPP will not be completely used, the connected area of WPP will be set as PQ node, and the power factor of WPP is within the reference bound. On the other hand, due to the reliable reactive power adjust ability of wind turbines under voltage control mode, the voltage in the wind injection area is more stable and the range of load margin is increased.

This change of voltage stability boundary can also be

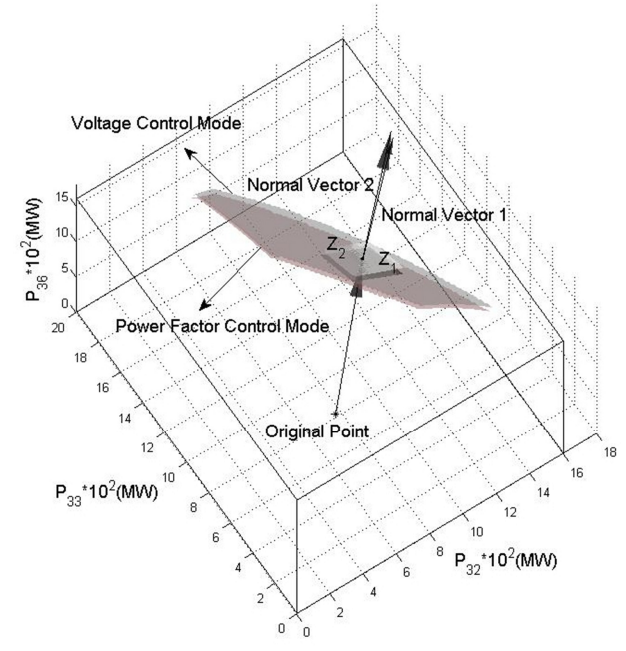

Fig. 8. Comparison figure of voltage stability boundary under different RPCMs.

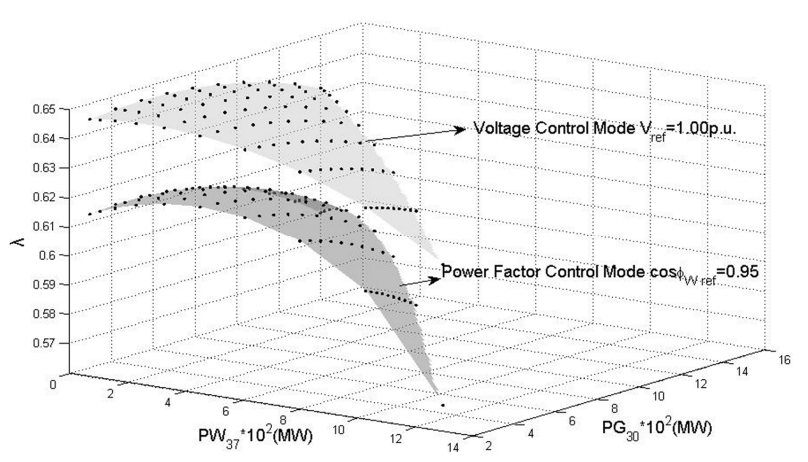

Fig. 9. Voltage stability boundary under different RPCMs in $\lambda-P W_{37}-P G_{30}$ space.

applied to enhance the loadability of power system with DFIG WPP, by switching WPP RPCM from power factor control mode to voltage control mode, and the voltage stability boundary can monitor this loadability change directly. According to the analysis, a reference operation strategy is offered. Stability boundaries of a WPP under different RPCMs are obtained beforehand in off-line analysis. When online monitoring, the operation security state of WPP in a certain wind condition can be calculated and estimated. It is a common case in practice that WPP 
Table 4. Calculation results of steady state parameters of DFIG WPP within voltage stability boundary under voltage control mode

\begin{tabular}{c|c|c|c|c|c|c|c}
\hline \multirow{2}{*}{ Wind Speed $(m / s)$} & \multicolumn{7}{|c}{ Parameters of DFIG WPP when $V_{\text {ref }}=1.00($ p.u. $)$} \\
\cline { 2 - 9 } & $Q_{W . c r t}(\mathrm{MW})$ & Vs(p.u.) & Vr.org-Vr.crt(p.u.) & Is.org-Is.crt(p.u.) & Ir.org-Ir.crt(p.u.) & $s($ p.u. $)$ & $\lambda($ p.u. $)$ \\
\hline $0-4,>21$ & $152.51-572.74$ & 1.0000 & $0.5861-0.5865$ & $0.0033-0.0112$ & $0.3478-0.3560$ & 0.5760 & 0.6334 \\
\hline 5 & $145.55-567.51$ & 1.0000 & $0.4782-0.4786$ & $0.0038-0.0112$ & $0.3477-0.3559$ & 0.4700 & 0.6336 \\
\hline 7 & $125.49-545.93$ & 1.0000 & $0.2626-0.2627$ & $0.0055-0.0116$ & $0.3473-0.3555$ & 0.2580 & 0.6342 \\
\hline 8 & $115.03-536.34$ & 1.0000 & $0.1547-0.1548$ & $0.0068-0.0122$ & $0.3472-0.3554$ & 0.1520 & 0.6342 \\
\hline 11 & $125.64-559.80$ & 1.0000 & $0.1689-0.1690$ & $0.0125-0.0164$ & $0.3476-0.3560$ & -0.1660 & 0.6296 \\
\hline 12 & $167.69-612.38$ & 1.0000 & $0.2767-0.2770$ & $0.0150-0.0188$ & $0.3485-0.3572$ & -0.2721 & 0.6244 \\
\hline $13-21$ & $250.18-716.09$ & 1.0000 & $0.3847-0.3850$ & $0.0179-0.0221$ & $0.3502-0.3593$ & -0.3781 & 0.6151 \\
\hline
\end{tabular}

Table 5. Calculation results of steady state parameters of DFIG WPP within voltage stability boundary under power factor control mode

\begin{tabular}{c|c|c|c|c|c|c|c}
\hline \multirow{2}{*}{ Wind Speed $(\mathrm{m} / \mathrm{s})$} & \multicolumn{7}{|c}{ Parameters of DFIG WPP when $\cos \varphi_{\text {Wref }}=0.95$} \\
\cline { 2 - 8 } & $Q_{W}(\mathrm{MW})$ & Vs.org-Vs.crt(p.u.) & Vr.org-Vr.crt(p.u.) & Is.org-Is. $\operatorname{crt}($ p.u. $)$ & Ir.org-Ir. $\operatorname{crt}($ p.u. $)$ & $s(\mathrm{p} . u)$. & $\lambda($ p.u. $)$ \\
\hline $0-4,>21$ & 0 & $0.9416-0.7876$ & $0.5517-0.4615$ & $0.0017-0.0020$ & $0.3249-0.2718$ & 0.5760 & 0.5911 \\
\hline 5 & 23.59 & $0.9486-0.7940$ & $0.4536-0.3797$ & $0.0027-0.0032$ & $0.3275-0.2743$ & 0.4700 & 0.5928 \\
\hline 7 & 64.72 & $0.9710-0.8172$ & $0.2549-0.2148$ & $0.0052-0.0062$ & $0.3360-0.2834$ & 0.2580 & 0.5979 \\
\hline 8 & 96.61 & $0.9860-0.8328$ & $0.1525-0.1289$ & $0.0068-0.0080$ & $0.3417-0.2892$ & 0.1520 & 0.6006 \\
\hline 11 & 251.14 & $1.0342-0.8682$ & $0.1747-0.1466$ & $0.0125-0.0149$ & $0.3608-0.3045$ & -0.1660 & 0.6008 \\
\hline 12 & 326.04 & $1.0444-0.8667$ & $0.2891-0.2399$ & $0.0149-0.0180$ & $0.3655-0.3055$ & -0.2721 & 0.5921 \\
\hline $13-21$ & 414.54 & $1.0440-0.8406$ & $0.4016-0.3235$ & $0.0177-0.0220$ & $0.3669-0.2987$ & -0.3781 & 0.5672 \\
\hline
\end{tabular}

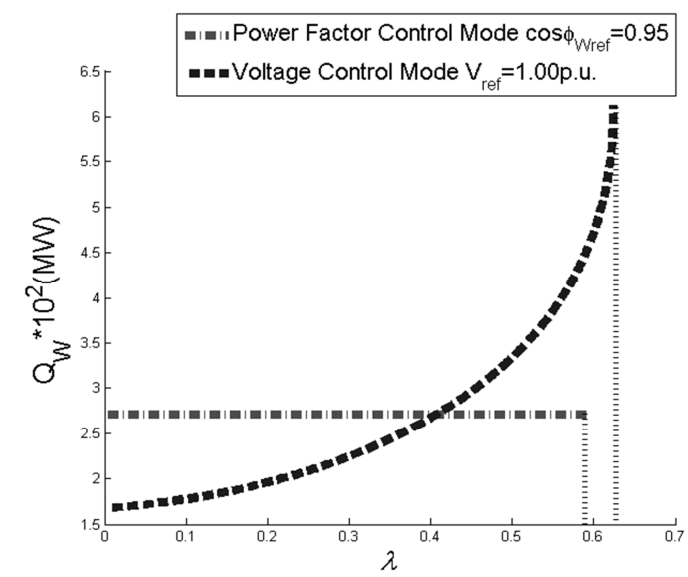

(a)

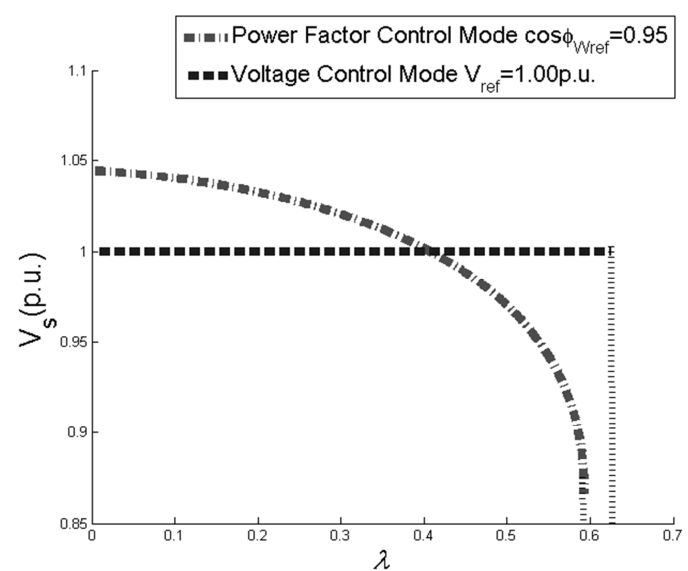

(b)

Fig. 10. Parameters of DFIG WPP under different RPCMs when wind speed is $12 \mathrm{~m} / \mathrm{s}$ : (a) Reactive power; (b) Stator voltage.

operates under power factor control mode, thus when online monitoring and calculation proves that the WPPconnected grid operates in a state fairly close to the stability boundary. With satisfying the active/reactive power limits of DFIG, it is feasible to switch RPCM to voltage control mode to promote system security.

For elaborating the impact of RPCM of DFIG WPP, the reactive power of DFIG WPP is analyzed in detail. To describe the regulation of load margin within boundary clearly, the steady state parameters of DFIG and the reactive power of WPP are analyzed below.

Stated in Table 4 and Table 5 are the steady state parameters of wind turbine and the reactive power of WPP under different WPP RPCM. No matter which kind of control mode the WPP is under, the steady state parameters, e.g., rotor voltage $V r$, stator current $I s$, rotor current $I r$ and slip $s$ change in the same way, and the effect on the loadability is slight.

However, the influence from reactive power is more obvious. When the WPP is under constant voltage control mode, the voltage of the bus is invariable at 1.0000 p.u., and the reactive power of WPP $Q_{W}$ is adjusted from 0Mvar to $720 \mathrm{Mvar}$ to balance the voltage fluctuation. To maintain the stability of area voltage, the converter of wind turbine is adjusting the reactive power unceasingly. On the contrary, when power factor control mode is adopted, the reactive power injection is less than that under the other control mode, and the voltage is decreased to the limit, e.g., 
the voltage of this area is dropped from 0.9860 p.u. to 0.8325 p.u., when wind speed is $8 \mathrm{~m} / \mathrm{s}$.

Fig. 10 shows the change trend of reactive power and stator voltage of DFIG wind turbine from original point to critical boundary. The maximum loadability $\lambda$ under constant voltage control mode is 0.6244 when the wind speed is $12 \mathrm{~m} / \mathrm{s}$ as well as the other loadability under the corresponding control mode is 0.5921 . From the curves, it can be summarized that the distinction of load margin under different control mode is mainly caused by the reactive power support of DFIG WPP. There is a rapid rise of WPP reactive power when the system is ahead to the boundary under voltage control mode. The value of $Q_{W}$ under voltage control mode is from $167.69 \mathrm{Mvar}$ to $612.38 \mathrm{Mvar}$, on the contrary, the $Q_{W}$ under power factor control mode remains constant. The increase of $Q_{W}$ can be interpreted that the reactive adjustment of DFIG voltage control can support the area stability and expand the load margin.

The voltage adjustment of wind injection area can increase the operation security recover capability and decrease the system collapse probability. When the reactive power of each DFIG wind turbine is adjusting the stability of this system, the other parameters of DFIG wind turbine have few contributions to keep the operation status stable.

\section{Discussion and Conclusion}

In this paper, the different RPCMs of DFIG WPP is investigated within voltage stability boundary. The analysis study of the simple system has expounded the difference between these two kinds of RPCMs in analytical derivation. The study of RPCM in large systems has also proposed a practical method to search the critical operation points under different RPCMs in multidimensional space.

From the work of analyses and simulations, the results of case study have illustrated that the loadability under voltage control mode is greater than the other one, which is in power factor control mode. It means that the voltage collapse point is harder to reach under voltage control mode and the voltage adjust ability is more reliable..

Furthermore, the critical point search method of this paper can provide a practical way for the stability judgment of power system with wind power injection, and the constructed voltage stability boundary can be also applied in system stability monitoring.

\section{Acknowledgements}

This work was supported by National Natural Science Foundation of China under Grant (51277015), Education Department Innovation Platform Open Foundation of Hunan Province(54291) and Graduate student research innovation project in Hunan province of China. (CX2015B364)

\section{Nomenclature}

\section{A. Variables}

E Electromotive force of bus 1 in the simple system.

$V \quad$ Voltage of bus 2 in the simple system.

$v \quad$ Wind speed.

$\sigma \quad$ Voltage angle of bus 2 in the simple system.

$\varphi_{W} \quad$ Power factor angle of WPP.

$\varphi_{L} \quad$ Power factor angle of load.

$P_{W} \quad$ Active power of DFIG WPP.

$Q_{W} \quad$ Reactive power of DFIG WPP.

$V_{\text {boundary }} \quad$ Voltage stability boundary of power system.

$P_{\text {L.max }} \quad$ Maximum loadability.

$f_{W} \quad$ Math model of DFIG WPP.

$f_{P} \quad$ Active power flow equations.

$f_{Q} \quad$ Reactive power flow equations.

$f_{C} \quad$ Reactive power control mode equations.

$V_{i} \quad$ Voltage of node $i$.

$\theta_{i} \quad$ Voltage angle of node $i$.

$V_{s}$ and $V_{r} \quad$ Stator and rotor voltages of wind turbine.

$\theta_{s}$ and $\theta_{r} \quad$ Stator and rotor voltage angles of wind turbine.

$I_{s}$ and $I_{r} \quad$ Stator and rotor currents of wind turbine.

$\phi_{s}$ and $\phi_{r}$ Stator and rotor current angles of wind turbine.

$s \quad$ Slip of wind turbine.

$\alpha_{i} \quad$ Increasing direction of generator node $i$.

$\beta_{i} \quad$ Increasing direction of load node $i$.

$\lambda \quad$ Load margin.

$\omega \quad$ Left eigenvector of Jacobian matrix.

$K_{G} \quad$ Generator space coefficients of tangent planes.

$K_{L} \quad$ Load space coefficients of tangent planes.

$K_{W} \quad$ Wind power space coefficients of tangent planes.

Coefficients of reactive power under voltage control mode.

\section{B. Constants}

$R_{m} \quad$ Magnetizing resistance of wind turbine.

$X_{m} \quad$ Magnetizing reactance of wind turbine.

$R_{s} \quad$ Stator resistance of wind turbine.

$X_{s} \quad$ Stator reactance of wind turbine.

$R_{r} \quad$ Rotor resistance of wind turbine.

$X_{r} \quad$ Rotor reactance of wind turbine.

$P_{\text {GB.Loss }} \quad$ Gear box power losses of wind turbine.

\section{Subscripts}

org Condition at original point.

crt Condition at critical point.

ref Reference value. 


\section{Sets}

$\begin{array}{ll}N_{L} & \text { Sets of load buses. } \\ N_{G} & \text { Sets of generators. } \\ N_{W} & \text { Sets of wind power injection buses. }\end{array}$

\section{References}

[1] E. Y. Bitar, R. Rajagopal, P. P. Khargonekar, K. Poolla, and P. Varaiya, "Bringing wind energy to market," IEEE Trans. Power Syst., vol. 27, no. 03, pp. 1225-1235, Aug. 2012.

[2] M. F. M. Arani and Y. A. I. Mohamed, "Analysis and impacts of implementing droop control in DFIG-based wind turbines on microgrid/weak-grid stability," IEEE Trans. Power Syst., vol. 30, no. 01, pp. 385-396, Jan. 2015.

[3] J. Kim, G. Park, J. K. Seok, B. Lee and Y.C. Kang, "Hierarchical voltage control of a wind power plant using the adaptive $\mathrm{I}_{\mathrm{Q}}-\mathrm{V}$ characteristic of a DoubleFed Induction Generator," J. Elect. Eng. \& Technol., vol. 10, no. 02, pp. 504-510, 2015.

[4] F. Wu and S. Kumagai, "Steady-state security regions of power systems," IEEE Trans. Circuits Syst., vol. 29, no. 11, pp. 703-711, Nov. 1982.

[5] S. Greene, I. Dobson, and F. L. Alvarado, "Sensitivity of the loading margin to voltage collapse with respect to arbitrary parameters," IEEE Trans. Power Syst., vol. 12, no. 01, pp. 262-272, Feb. 1997.

[6] C. Wang, M. Fan, and W. Wei, "Research on voltage stability security region in area total load space," Proc. CSEE, vol. 29, no. 8, pp. 62-67, Oct. 2009.

[7] N.V. Thang, N. Minh Y, Y. T. Yoon, "A new method for monitoring local voltage stability using saddle node bifurcation set in two dimensional power parameter space," J. Elect. Eng. \& Technol., vol. 08, no. 02, pp. 206-214, 2013.

[8] C. Wang, X. Wang, and W. Sun, "Fast calculation and analysis of probabilistic total transfer capability in power system including large-scale WPPs," Proc. CSEE, vol. 28, no. 10, pp. 56-62, Apr. 2008.

[9] W. Miao and H. Jia, "An innovative dispatching, monitoring, and control method for large-scale WPP integration," in Proc. 2012 IEEE Power and Energy Society General Meeting, San Diego, CA.

[10] Y. Mu and H. Jia, "An approach to determining the local boundaries of voltage stability region with WPPs in power injection space," Sci. China Ser. Etech. Sci., vol. 53, no. 12, pp. 3232-3240, Dec. 2010.

[11] J. Zhao, X. Li, J. Hao, and J. Lu, "Reactive power control of WPP made up with doubly fed induction generators in distribution system," Electric Power System Research, vol. 80, no. 06, pp. 698-706, Jun. 2010.
[12] A. Mousavi, M. Bozorg, and R. Cherkaoui, "Preventive reactive power management for improving voltage stability margin," Elect. Power Syst. Res., vol. 96, pp. 36-46, Mar. 2013.

[13] D. Santos-Martin, S. Arnaltes, and J. L. R. Amenedo, "Reactive power capability of doubly fed asynchronous generators," Elect. Power Syst. Res., vol. 78, no. 11, pp. 1837-1840, Nov. 2008.

[14] R. S. Al Abri, E. F. El-Saadany, and Y. M. Atwa, "Optimal placement and sizing method to improve the voltage stability margin in a distribution system using distributed generation," IEEE Trans. Power Syst., vol. 28, no. 01, pp. 326-334, Feb. 2013.

[15] J. Padrón and A. Lorenzo, "Calculating steadyState operating conditions for doubly-fed induction generator wind turbines," IEEE Trans. Power Syst., vol. 25, no. 2, pp. 922-928, May. 2010.

[16] M. Kay and J. V. Milanovi'c, "Reactive power control strategies for DFIG-based plants," IEEE Trans. Energy Convers., vol. 22, no. 02, pp. 389-396, Jun. 2007.

[17] H. Ko, G. Yoon, and W. Hong, "Active Use of DFIGBased Variable-Speed Wind-Turbine for Voltage Control in Power System Operation," J. Elect. Eng. \& Technol., vol. 03, no. 02, pp. 254-262, 2008.

[18] Y. Z. Lin, L. B. Shi, L. Z. Yao, Y. X. Ni, S. Y. Qin, R. M. Wang and J. P. Zhang, "An analytical solution for voltage stability studies incorporating wind power," $J$. Elect. Eng. \& Technol., vol. 10, no. 03, pp. 865-876, 2015.

[19] G. Tapia, A. Tapia, and J. X. Ostolaza "Proportionalintegral regulator-based approach to WPP reactive power management for secondary voltage control," IEEE Trans. Energy Convers., vol. 22, no. 02, pp. 389-396, Jun. 2007.

[20] Q. Guo, H. Sun, B. Wang, B. Zhang, W. Wu, and L. Tang "Hierarchical automatic voltage control for integration of large-scale wind power: Design and implementation," Elect. Power Syst. Res., vol. 120, no. 03, pp. 234-241, Mar. 2015.

[21] C. Hamon, M. Perninge, and L. Söder, "A stochastic optimal power flow problem with stability constraintspart I: approximating the stability boundary," IEEE Trans. Power Syst., vol. 28, no. 02, pp. 1839-1848, May. 2013.

[22] M. Perninge and L. Söder, "On the validity of local approximations of the power system loadability surface," IEEE Trans. Power Syst., vol. 26, no. 04, pp. 2143-2153, Nov. 2011.

[23] Z. Qin and R. Ma, "Research on voltage stability region tangent plane of power system with doublyfed induction generator WPP," in Proc. 2014 IEEE Power and Energy Society General Meeting, Washington, DC. 


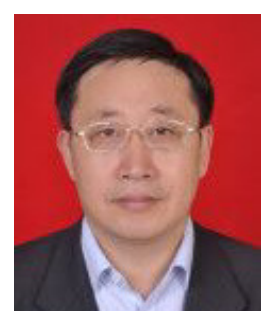

Rui Ma He received the B.Sc. degree in Electrical Engineering, the M.Sc. degree in Control Theory and Control Engineering, and the Ph.D. degree in Electrical Engineering from Changsha University of Electric Power, Hunan University and North China Electric Power University in 1994, 1999 and 2006, respectively. From September 2009 to July 2011, He was a Visiting Scholar in Texas A\&M University. Now he is a Professor in the School of Electrical and Information Engineering, Changsha University of Science and Technology. His research is in the area of power system stability analysis, renewable energy accessing, electrical market and low-carbon electricity.

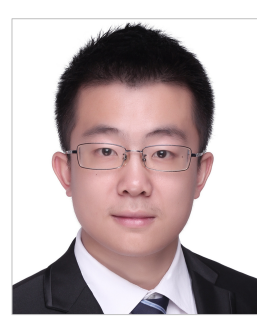

Zeyu Qin He received the B.Sc. degree in Electrical Engineering from Changsha University of Science and Technology in 2013. He is currently pursuing the M.Sc. degree in the same University. His research interests include power system stability analysis and low-carbon electricity.

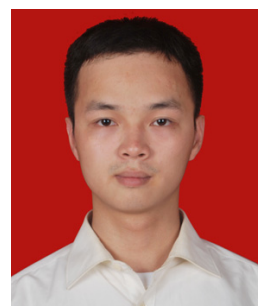

Wencan Yang $\mathrm{He}$ received his bachelor's degree from Changsha University of Science and Technology in 2014. He is currently working towards the master degree in the same University. His research interests are wind power planning and power system stability analysis.

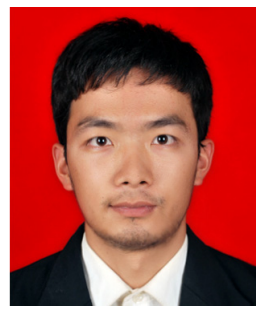

Mo $\mathbf{L i ~ H e ~ r e c e i v e d ~ t h e ~ B . S c . ~ d e g r e e ~ i n ~}$ Electrical Engineering from Hunan Institute of Engineering in 2013. He is currently pursuing the M.Sc. degree in Changsha University of Science and Technology. His research interests include operation and control of power system and power system stability

analysis. 